\title{
Study of the Pulsed Field Magnetization Strategy for the Superconducting Rotor
}

\author{
Zhen Huang, H. S. Ruiz, Jianzhao Geng, Boyang Shen, and T. A. Coombs
}

\begin{abstract}
High magnetic field can be trapped in a bulk type-II superconductor through different magnetization methods. In our fully high temperature superconducting (HTS) synchronous motor, the pulsed field magnetization (PFM) method is adopted due to its reduced cost, feasibility, and adaptability on the assembling of our HTS motor system. The HTS rotor was designed with seventy-five YBCO HTS bulks mounted on the surface of the rotor to make two pairs of poles after the PFM. In this paper, we give a PFM circuit used to generate a pulsed magnetic field about $1.5 \mathrm{~T}$ at the centre of the magnetizing coil lasting less than $1 \mathrm{~s}$ by discharging a capacitor bank. We also investigate the homogeneity of the pulsed magnetic field produced by the coils. The main strategy for magnetizing the superconducting rotor is designed and investigated and the rotor field profile after the whole magnetization procedure is measured and discussed as well.
\end{abstract}

Index Terms - HTS synchronous motor, HTS rotor, pulsed field magnetization.

\section{INTRODUCTION}

$\mathrm{H}$ IGH magnetic fields can be trapped in bulk type-II superconductors through different magnetization methods. Normally, there are four types of magnetization methods: fieldcooled (FC) magnetization [1], zero-field-cooled (ZFC) magnetization, pulsed field magnetization (PFM) [2, 3, 4], and flux pumping $[5,6]$. In the FC method the sample is cooled below its critical temperature in the presence of a strong DC magnetic field which is subsequently removed. In the ZFC and PFM methods the HTS bulk is first cooled below its critical temperature, and then an external magnetic field which increases from zero to a peak value and then decreases to zero is applied to magnetize the bulk superconductor. However, the disadvantage of ZFC is that it needs a bigger coil which is used to produce a strong magnetic field for a relatively long time in comparison with PFM. Hence the cost of the coil for the ZFC is considerably high if a large magnetic field (e.g. 5T) is required. However, the coil used for PFM is cheap because less copper is required as the current during the PFM is transient. Therefore, it becomes the most practical method. The pulse time of the PFM is of the order of milliseconds. However, the rapid changes in magnetic field making heat generation by the PFM are significant, possibly causing a reduction in the trapped magnetic field of the superconducting bulk due to the decrease in its critical current density. In the flux pumping a field is swept across the superconductor in a magnetic wave. As long as the direction of motion of the magnetic wave is constant, then

Manuscript received October 18, 2015.

Zhen Huang and H. S. Ruiz were with the Electrical Engineering Division, Department of Engineering, University of Cambridge, CB3 OFA, Cambridge, UK. Zhen Huang is now with the Department of Electrical Engineering, Shanghai Jiao Tong University, 800 Dongchuan Road, Minhang District, Shanghai, China, e-mail: zh252@cam.ac.uk. H. S. Ruiz is now with the

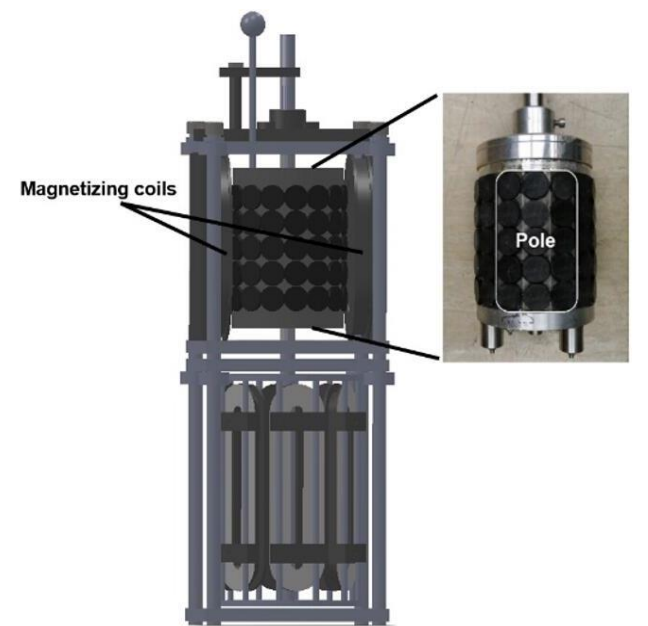

Fig. 1. Illustrative picture of the prototype of our fully HTS synchronous motor developed at the University of Cambridge. A real picture of the rotor, $220 \mathrm{~mm}$ height, $85 \mathrm{~mm}$ radius (including the $5 \mathrm{~mm}$ thickness of the HTS bulk) is shown in the inset. Seventy-five polished YBCO rounded bulks have been mounted on the rotor, in order to cover its entire surface area as shown in the picture. Four effective poles are considered, such that each pole is composed by three columns of bulks as shown in the inset.

the current induced will always move in the same direction. Successive waves will induce more and more current within the bulk superconductor and higher magnetic fields will be trapped. Any of these methods could be used to magnetize the superconductor. In our fully HTS synchronous motor, the PFM method is adopted because it is cheap, feasible, and facilitates the assembling of our HTS motor system.

This paper is organized as follows: In Section II, design parameters and experimental setup of the PFM system with emphasis on the rotor structure are described. Then, in Section III, we explain the circuit design constructed to perform the PFM of the HTS rotor. Hence, in Section IV our experimental results about the different pulsed fields and the rotor surface field profile after the PFM, are presented and discussed. Finally, in Section V we summarize the main conclusions of this study.

\section{PFM SYSTEM DESIGN AND EXPERIMENTAL SETUP}

The superconducting rotor is shown in Figure 1. Seventy-five polished YBCO bulks of rounded shape, $31 \mathrm{~mm}$ diameter and $5 \mathrm{~mm}$ thickness, are mounted on the surface of the rotor. The bulk array contains fifteen columns and each column has five pieces. The white box in the inset of Figure 1 illustrates a typical pole of the designed HTS rotor. The height (h) and radius (r) of

Department of Engineering, University of Leicester, University Road, LE1 7RH, Leicester, UK, email: dr.harold.ruiz@leicester.ac.uk.

Jianzhao Geng, Boyang Shen, and T. A. Coombs are with the Electrical Engineering Division, Department of Engineering, University of Cambridge, CB3 0FA, Cambridge, UK, email: tac1000@cam.ac.uk 


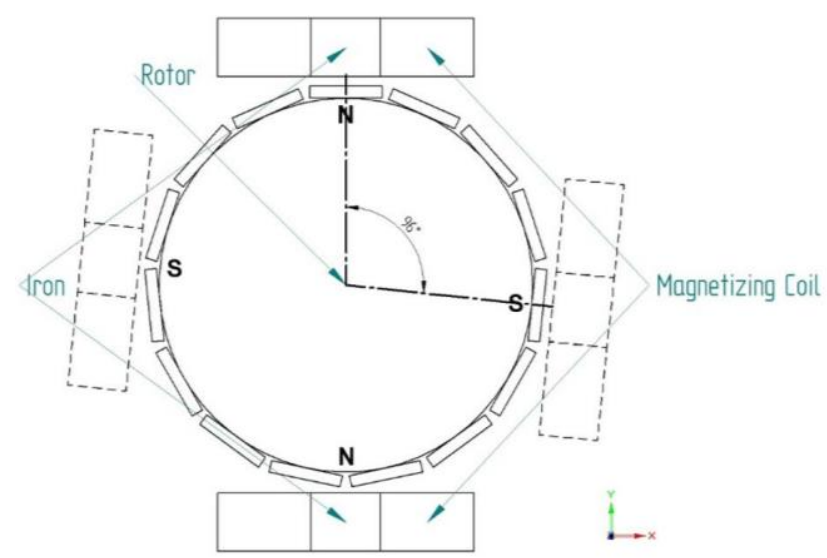

Fig. 2. Schematic diagram of the PFM system which shows the relative positions between the magnetizing coils and bulks. The magnetizing coils drawn by continuous line indicate the first pulse position and, the magnetizing coils drawn by dashed lines indicate the second pulse position after a rotation of $96^{\circ}$ as explained in the text.

this rotor are $220 \mathrm{~mm}$ and $80 \mathrm{~mm}$, respectively. There is a pair of magnetizing copper coils installed on the top of the motor rig. One pair of copper coils mounted on the stainless frame is used to magnetize the rotor through the PFM. The setup of the magnetising coils is displayed in Figure 1. The width between these two coils is $17.8 \mathrm{~cm}$ and the coils are connected in series. An iron core is embedded in the middle of the coil, concentrating the magnetic flux during the PFM and enhancing the magnetic field produced by the coil dramatically. Fourhundred and seventy-seven turns of copper wire for each coil are wound on the former of the coil. The inductance and resistance of the coil are $87.6 \mathrm{mH}$ and $0.6 \Omega$ (at $77 \mathrm{~K}$ ), respectively. During the magnetization procedure, a rotor whose diameter is $170 \mathrm{~mm}$ is put in the middle of two coils, so that the gap between coil and rotor is $4 \mathrm{~mm}$ on each side.

In figure 2, a schematic diagram of the PFM system is displayed. In the middle of the coils there is a rotor with fifteen columns of YBCO bulks to make a four-pole machine after the PFM. Before the experiment, the bulks are cooled down below their critical temperature $(92 \mathrm{~K})$ using liquid nitrogen at $77 \mathrm{~K}$. The process of the magnetization is as follows: firstly, magnetic pulses are applied to the centre of one column bulks to produce a pair of north $(\mathrm{N})$ poles. Secondly, the armature of the rotor is rotated by using an assembled steering wheel with a precision universal protractor $\left(1 / 5^{\circ}\right)$ which has the same axle as the rotor such that a second group of bulks is magnetized in order to produce a pair of south (S) poles. Prior to performing the PFM, the angular position of the rotor is adjusted carefully in order to ensure that the pulsed field is orthogonal to the bulks' faces. The rotor is clamped firmly to prevent rotation by the relatively strong magnetic torque generated by the pulsed field during the magnetization procedure. When making $\mathrm{S}$ poles, the rotor rotates $96^{\circ}$ instead of $90^{\circ}$ (Figure 2). This is because in order to achieve the maximum magnetization of the pole, the second pulse position has to be strictly aligned with the centre of one column of bulks rather than in between two successive columns.

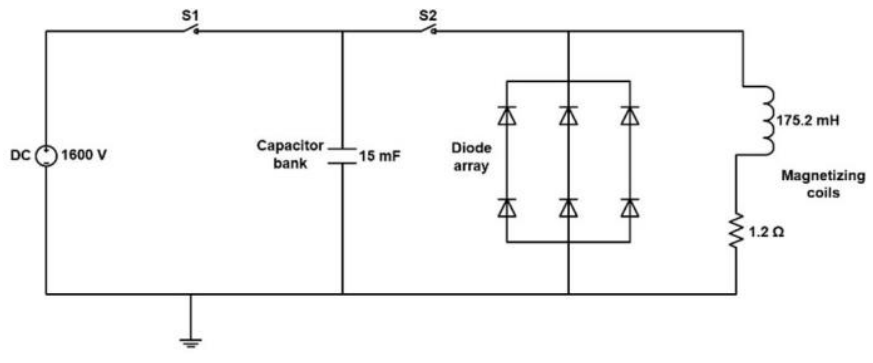

Fig. 3. Schematic diagram of the pulse circuit designed for the PFM

It is worth mentioning that the number of HTS bulk columns used to cover the surface of the rotor is actually a compromise between the dimensions of the rotor itself, the dimensions of the superconducting bulks, and associated costs. On the one hand, the curvature of the rotor create constrictions on the size and manufacturing of the SC bulks for being attached to the surface of the rotor. Furthermore, during and after the PFM the SC bulks are subjected to a significant magnetic stress which compromise their mechanical structurality, reason why during the prototyping phase of this project, we have reduced the size of the SC bulks in order to homogeneously cover the entire peripheral surface of the rotor. However, given the fact that a reduction of costs is vital for the proof of concept of the prototype, in order to minimize the overall costs of the motor without compromising the initial aims of this project, the rotor structure has been taken from a previous flywheel project [7], which has resulted in fifteen columns of HTS bulks of $31 \mathrm{~mm}$ diameter each, which allows the effective magnetization of at least 12 columns for the creation of four poles with 3 columns each as shown in Figs. 1 \& 2. Nevertheless, this configuration (odd number of columns) will make the field distribution slightly asymmetric, i.e. effectively, two poles will be seen as containing odd numbers of columns of bulks, with the other two poles containing an even number. This effect will be shown in Section IV from our experimental measurements.

TABLE I

CHARACTERISTIC PARAMETERS IN THE PFM CIRCUIT

\begin{tabular}{lc}
\hline \hline \multicolumn{1}{c}{ Parameters } & Value \\
\hline Discharging current & $375 \mathrm{~A}$ \\
Charging voltage & $1.6 \mathrm{kV}$ \\
Charging current & $120 \mathrm{~mA}$ \\
Induction of coils & $2 \times 87.6 \mathrm{mH}$ \\
Resistance of coils & $2 \times 0.6 \Omega(\mathrm{at} 77 \mathrm{~K})$ \\
Capacitance bank & $15 \mathrm{mF}$ \\
Central magnetic field & $1.5 \mathrm{~T}$ \\
Pulse width & $0.8 \mathrm{~s}$ \\
\hline These parameters are relative to the circuit design illustrated in Fig. 3
\end{tabular}

\section{PFM CIRCUIT DESIGN}

A PFM circuit is designed to generate a pulsed magnetic field lasting less than $1 \mathrm{~s}$ by discharging a capacitor bank. The objective of this design is to apply a pulsed current of around $400 \mathrm{~A}$ (this is limited by the thickness of the copper wire) in two serially connected copper coils, in order to generate a pulsed magnetic field about $1.5 \mathrm{~T}$ at the centre of the magnetizing coil. Also, the pulses are repeatable. A schematic 

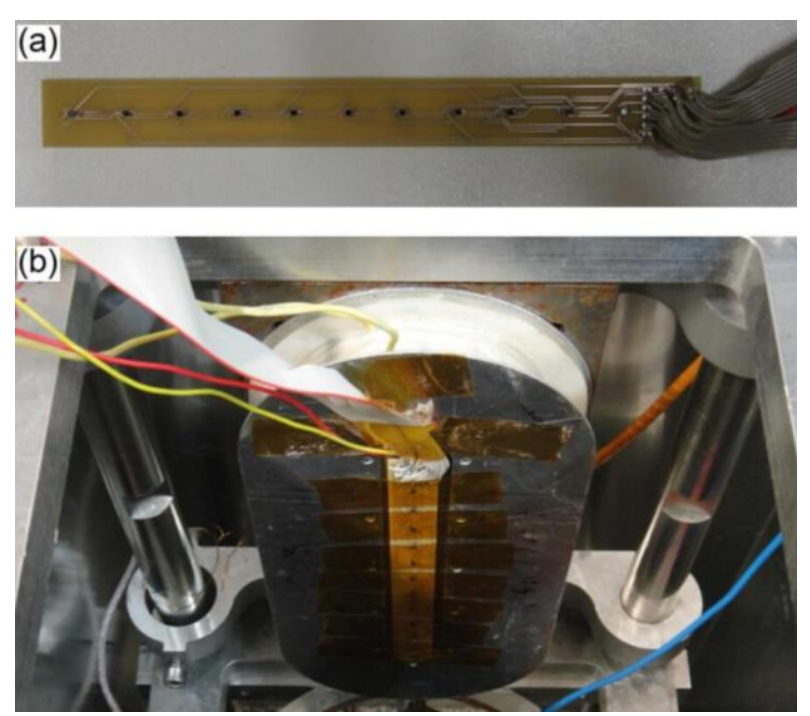

Fig. 4. PCB and its setup for measuring the magnetic fields produced by the magnetizing coil. In (a) the 11 hall probes with relative separation of about $16.5 \mathrm{~mm}$ are shown. In (b) the final position of the PCB is shown, it at the centre of the magnetizing coil.

diagram of the pulse circuit is presented in Figure 3. According to this circuit, there are two steps to perform the PFM. First, the switch S1 is turned on whilst the switch S2 is kept turned off. A large capacitor bank which can provide a high pulsed current to the coils is adopted. Then, when the charging of the capacitor is completed, the switch $\mathrm{S} 1$ is turned off and the switch $\mathrm{S} 2$ is turned on, it in order to discharge the capacitor. It is worth noting that six diodes are connected to the circuit to prevent electrical resonance. The two magnetizing coils are modelled as an equivalent inductor and resistor. Furthermore, three capacitors of $1500 \mathrm{~V}$ and $5 \mathrm{mF}$ each, are connected in parallel, such that the total capacitance is of $15 \mathrm{mF}$. The main parameters in the PFM circuit are summarized in Table 1.

\section{EXPERIMENTAL RESULTS AND DISCUSSIONS}

After the electronic circuits and power electronics have been prepared and properly set up, the printed circuit board (PCB) shown in Figure 4(a), which includes eleven hall probes, was made for the experiments presented in this section.

The dimensions of this PCB are $192 \mathrm{~mm}$ length, $25 \mathrm{~mm}$ width, and $2 \mathrm{~mm}$ thickness. All the hall probes are of the HZ$312 \mathrm{C}$ type (active surface: $2.35 \mathrm{~mm} \times 2.7 \mathrm{~mm}$ ), produced by Asahi Kasei, and are connected in series. The average interval between each hall probe is about $16.5 \mathrm{~mm}$ and all hall probes are calibrated beforehand. Then, before performing the experiment, the PCB is placed at the centre of the magnetizing coil and fixed using Kapton tape to make sure that the surfaces of the hall probes are parallel to the magnetizing coil (see Fig. 4 (b)). It allows to measure the magnetic induction perpendicular to the magnetizing coil. One triggered current source which can stably provide a $5 \mathrm{~mA}$ current is used to supply the current to the hall probes and the data are acquired using National Instruments PCI-6221.

Figure 5 shows the pulsed field data recorded by the middle of the hall probe, which shows the corresponding magnetic fields produced at the centre of one magnetizing coil after

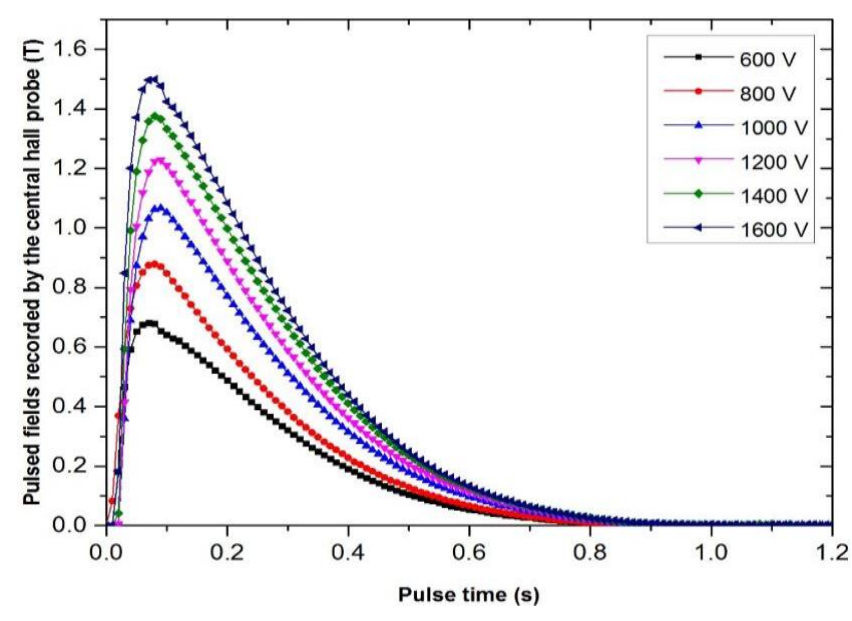

Fig. 5. Experimental results of pulsed magnetic fields produced at the centre of the magnetizing coil under different charging voltages ranging from $600 \mathrm{~V}$ to $1600 \mathrm{~V}$.

immersing it in LN2 under different discharging voltages. The rise time is $0.07 \mathrm{~s}$ and pulse width is $0.8 \mathrm{~s}$. According to the experiment, when the discharging voltage reaches $1600 \mathrm{~V}$, the peak of the pulsed magnetic field is $1.5 \mathrm{~T}$. Figure 6 depicts the implemented $2 \mathrm{~mm}$ away from the coil because of the thickness of pulsed magnetic fields along the centre of the magnetizing coil, which is recorded by all eleven hall probes. The measurement is the PCB and cubic interpolation is used when plotting this graph. According to this graph, the magnetizing coils are able to produce a relatively homogeneous field in the central column due to the presence of the iron core. When the pulsed field reaches the maximum peak $(0.07 \mathrm{~s})$, the field at the edges is slightly higher than the field in the middle and the difference is around $100 \mathrm{mT}$. As this is the field which the bulks will experience directly, it is better to make the field as homogeneous as possible and the result for our coil is acceptable.

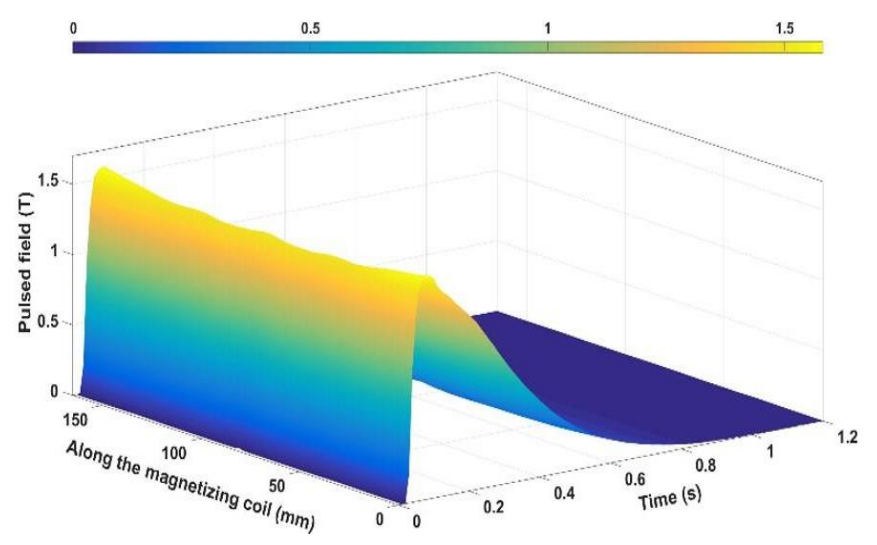

Fig. 6. Experimental results of the pulsed magnetic fields along the centre of the magnetizing coil when the charging voltage is $1600 \mathrm{~V}$.

According to the PFM strategy described in Section II, and illustrated in Figure 2, we first align one column of bulks to face the magnetizing coil and then, several pulses are applied in order to let to the bulks trapping greater magnetic fields. For each pulse, we wait for about $5 \mathrm{~min}$ to charge the capacitor to $1600 \mathrm{~V}$ and then, after discharging, each measurement is 


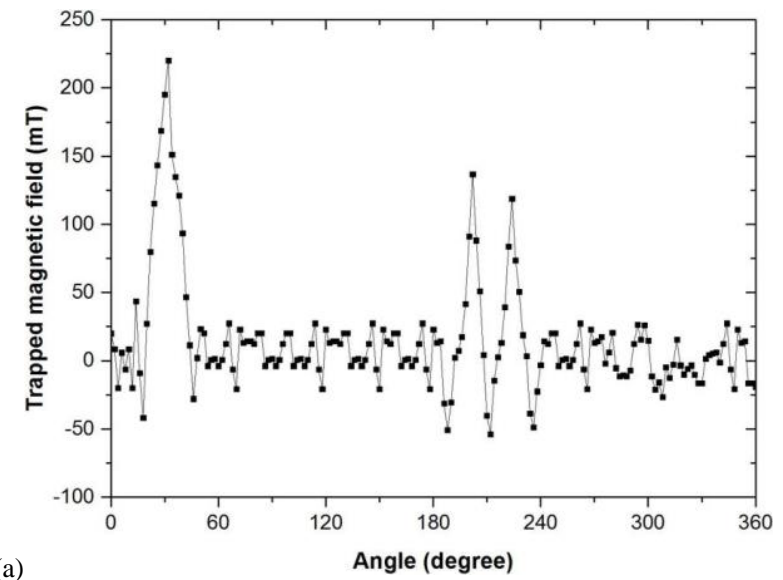

(a)

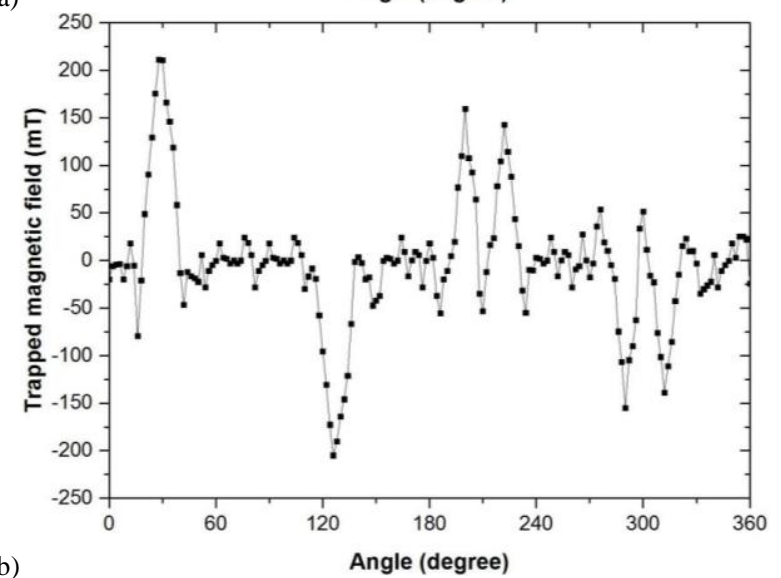

(b)

Fig. 7. (a) $360^{\circ}$ field profile measured $4 \mathrm{~mm}$ away from the rotor surface after three magnetization pulses. (b) same as above, but after the six pulses procedure, i.e., three pulses are initially applied to produce the $\mathrm{N}$ poles, and then another three pulses are applied to produce the $\mathrm{S}$ poles of the rotor.

recorded $10 \mathrm{~min}$ after the completion of the PFM in order to avoid the influences of magnetic flux creep. The time interval between applying two successive pulses is roughly $15 \mathrm{~min}$.

The PCB shown in Figure 4(a) is sealed and protected by wrapping it with Kapton tape before inserting the rotor into the rig for the experiments, which may damage the tin on the PCB. There are eleven hall probes on the $\mathrm{PCB}$, which are positioned at the bulks' centres and edges. According to [8], the bulks can trap about $89 \%$ of the maximum trapped field after three pulses.

Therefore, after balancing the magnetization time and the trapped magnetic fields' levels, only three pulses are used in all subsequent experiments. Moreover, when applying more pulses, the bottom and top bearings sometimes freeze, and then the experiment cannot go any further. For instance, if we apply seven pulses this takes about $105 \mathrm{~min}$, which is too long for the magnetization procedure and therefore the bearings are very likely to freeze.

In figures 7 (a) and (b), we present 180 sets of data each, which have been recorded by the middle hall probe for $360^{\circ}$ ( $2 \%$ sample). Figure 7 (a) illustrates that the rotor can trap a pair of $\mathrm{N}$ poles after three PFM repetitions. The $\mathrm{x}$ axis gives the angular position and the $y$ axis gives the trapped magnetic fields on the surface of the rotor. According to this figure, the $\mathrm{N}$ pole has trapped $220 \mathrm{mT}$ peak magnetic field. The trapped field shapes are not completely symmetrical due to the inherent experimental errors in the rotating procedure, as well as due to manufacturing, aging, and/or physical inhomogeneities in the superconducting bulks. The rotor pole contains three columns of bulks but only the central one is affected by the PFM. Therefore, this pole formed by a cylinder bulk array $(1 \times 5)$ gives a total magnet area of approximately $50.5 \mathrm{~cm}^{2}(31 \mathrm{~mm} \times 163$ $\mathrm{mm})$. The other $\mathrm{N}$ pole is roughly at the position of $216^{\circ}$. This pole is different from the previous $\mathrm{N}$ pole due to the odd numbers of columns being magnetized. On this side, the pulsed magnetic fields are applied between two columns of bulks as illustrated in Figure 2. Therefore, according to Figure 7, two columns of the bulks have been magnetized. The trapped magnetic fields of these two columns are lower than the other $\mathrm{N}$ pole because the applied magnetic field is not perpendicular to the surface of the bulks, i.e. there are more than one component of the magnetization current inside the bulk after the PFM, in order to produce normal and tangential magnetic field. The peak magnetic field of the $\mathrm{N}$ pole at $36^{\circ}(220 \mathrm{mT})$ is 1.7 times larger than the average peak magnetic field of the $\mathrm{N}$ pole at $216^{\circ}(127.7 \mathrm{mT})$. However, the total maximum magnetic field of the $\mathrm{N}$ pole at $36^{\circ}(220 \mathrm{mT})$ is $86 \%$ of the total maximum magnetic field of the $\mathrm{N}$ pole at $216^{\circ}(255.4 \mathrm{mT})$, which is greater, because there are two columns of magnetized bulks.

Thus, after finishing the measurement, the rotor is rotated anticlockwise a further $6^{\circ}$ in order to let the centre of one column of bulks at $96^{\circ}$ face to the magnetizing coil again, as indicated in Figure 2. The capacitor's polarity is reversed (i.e. the direction of the pulsed field changes) by swapping the charging cables in order to magnetize the bulks as a pair of Spoles. Figure 8 shows the field profile after a total of six pulses and after each pulse, they all have a 10 min magnetic relaxation time. This field profile proves that the rotor can become a fourpole machine after the PFM procedure is completed.

By comparing Figs. 7 (a) and (b), it is possible to observe that the last three pulses do not affect the trapped fields on the previously magnetized bulks. However, due to the odd number of columns, one $\mathrm{N}$ pole at $216^{\circ}$ and one $\mathrm{S}$ pole at $312^{\circ}$ contain both two columns of bulks which have been magnetized, and each one of the bulks on these two poles trap less magnetic field compared with the bulks on the other two corresponding poles which only show one column of magnetized bulks. The peak values for the $\mathrm{N}$-pole at $36^{\circ}$ and $\mathrm{S}$-pole at $126^{\circ}$ are of $211.3 \mathrm{mT}$ and $204.9 \mathrm{mT}$, respectively. However, the intensity of trapped magnetic fields could be dramatically enhanced by using state of the art bulk materials as it has been shown in Ref. [9].

\section{CONCLUSION}

The PFM method has been performed in order to magnetize the rotor of our prototype for the development of a fully HTS motor. A peak field of $220 \mathrm{mT}$ can be trapped by the HTS bulks in order to maintain the electromagnetic torque. Varying pulsed magnetic fields produced at the centre of one magnetizing coil under different discharging voltages, ranging from $600 \mathrm{~V}$ up to $1600 \mathrm{~V}$ have been tested. The rise time of the pulsed field is $0.07 \mathrm{~s}$ and pulse width is $0.8 \mathrm{~s}$. According to the experiment, 
when the discharging voltage reaches $1600 \mathrm{~V}$, the peak of the pulsed magnetic field is $1.5 \mathrm{~T}$. Eleven hall probes are used to record the pulsed magnetic fields along the centre of the magnetizing coil. The experimental results prove that the magnetizing coils are able to produce a relatively homogeneous field in the central column due to the presence of the iron core. Also, the field profile measured after the whole PFM procedure shows that the rotor can become a four-pole machine by implementing the current magnetization strategy. However, although at this stage a proof of concept has been successfully validated, this prototype presents some issues concerning to the optimal use of the PFM strategy. Firstly, our current strategy only takes advantage of six columns of bulks because of the size of the magnetizing coils and their iron core. At this stage, this strategy has been employed only for the sake of simplicity, and under the scope of a proof of concept. However, in future tests the inefficient use of bulks on the rotor will be overcome by the sequential magnetization of the individual columns that conform each one of the poles. Secondly, because the relative dimensions between the rotor and the HTS bulks, in the current prototype there is an asymmetric distribution of the trapped field due to the resulting odd number of columns. Therefore, thanks to the gained experience a more optimal design of the rotor dimensions and bulks will be considered in the future. On the other hand, by using state of the art HTS bulks, also a significate enhancement on the intensity of trapped magnetic field is to be expected.

\section{ACKNOWLEDGMENT}

The authors express their appreciation to Dr. Koichi Matsuda, Dr. Zhaoyang Zhong, Dr. David Hasko, Fred Spaven, and John Grundy for their valuable help and discussions.

\section{REFERENCES}

[1] M. Koblischka, T. Johansen, and M. Murakami, "Field-cooled magnetization

measurements of nd-123 bulk superconductors," Superconductor Science and Technology, vol. 13, no. 6, p. 745, 2000.

[2] H. Fujishiro, M. Kaneyama, T. Tateiwa, and T. Oka, "Record-high trapped magnetic field by pulse field magnetization using gdbacuo bulk superconductor," Japanese journal of applied physics, vol. 44, no. 9L, p. L1221, 2005.

[3] H. Fujishiro, T. Tateiwa, A. Fujiwara, T. Oka, and H. Hayashi, "Higher trapped field over $5 \mathrm{t}$ on htsc bulk by modified pulse field magnetizing," Physica C: Superconductivity, vol. 445, pp. 334-338, 2006.

[4] H. Fujishiro, T. Hiyama, T. Miura, T. Naito, S. Nariki, N. Sakai, and I. Hirabayashi, "Pulsed field magnetization for gdbacuo bulk with stronger pinning characteristics," Applied Superconductivity, IEEE Transactions on, vol. 19, no. 3, pp. 3545-3548, 2009.

[5] T. Coombs, Z. Hong, and X. Zhu, "A thermally actuated superconducting flux pump," Physica C: Superconductivity, vol. 468, no. 3, pp. 153-159, 2008.

[6] Q. Li, Y. Yan, C. Rawlings, and T. Coombs, "Numerical analysis of thermally actuated magnets for magnetization of superconductors," in Journal of Physics: Conference Series, vol. 234, p. 032035, IOP Publishing, 2010.

[7] T. A. Coombs, A. Cansiz, and A. M. Campbell, "A superconducting thrust-bearing system for an energy storage flywheel," Superconductor Science and Technology, vol. 15, no. 5, pp. 831-835, 2002.

[8] Z. Huang, M. Zhang, W. Wang, and T. A. Coombs, "Trial test of a bulktype fully HTS synchronous motor," Applied Superconductivity, IEEE Transactions on, vol. 24, no. 3, pp. 1-5, 2014.
[9] J. H. Durrell, A. R. Dennis, J. Jaroszynski, M. D. Ainslie, K. G. Palmer, Y-H Shi, A. M. Campbell, J. Hull, M. Strasik, E. Hellstrom, and D. A. Cardwell, "A trapped field of $17.6 \mathrm{t}$ in melt-processed, bulk gd-ba-cu-o reinforced with shrink-fit steel," Superconductor Science and Technology, vol. 27, no. 8, p. 082001, 2014. 